\title{
The Versatility of Temporalis Muscle Flap in Reconstruction of Maxillofacial Region Defects
}

\author{
AHMAD S. ${ }^{1}$, WARRAICH R.A. ${ }^{2}$, ABID H., ALI S. ${ }^{3}$ \\ Address for Correspondence: Dr Shakeel Ahmad, Assistant Prof. Oral Surgery Department, University College of Dentistry, \\ the University of Lahore
}

Introduction: The temporalis muscle flap is an axial pattern flap based on the anterior and posterior deep temporal arteries. It is most commonly utilized after maxillectomy. The temporalis flap splits into anterior and posterior halves, or the entire muscle used as a flap.

Objectives: The purpose of this study was to: 1 . Evaluate the esthetic and functional outcome by using temporalis muscle flap for reconstruction of intraoral defect. 2. Assess the effectiveness of temporalis muscle flap in intraoral defects.

Study Design: It was a Descriptive study

Setting: The study carried out at department of Oral and Maxillofacial Surgery King Edward Medical University / Mayo Hospital Lahore.

\section{Subjects and Methods}

Duration: The study was completed in six months from $1^{\text {st }}$ June 2005 to $30^{\text {th }}$ Nov 2005.

Sample size: 30 patients who presented with intraoral defect were included in this study.

Sample Technique: Nonprobability Sampling (Purposive).

Results: Success rate was ninety percent in this study. Failure of flaps encountered in $10 \%$ cases especially in older and immunocompromised patients.

Conclusions: Temporalis muscle flap is a good choice for most of the intraoral defects especially those in palatal and buccal region.

Key Words: Temporalis muscle flap, Palatal reconstruction, oral cavity, Mucosalisations.

\section{Introduction}

Reconstruction should strive to restore the maxillofacial form, versatility of tissues, oral competence, and oral cavity functions and thus allowing the patients to meet the social needs. Temporalis muscle flap holds great promise for the reconstruction of various maxillofacial region defects ${ }^{1}$. This flap is an axial pattern based on the anterior and posterior deep temporal arteries ${ }^{2}$. Flap covering solid structures as bone or reconstruction plates adapts well to the transplant bed $^{3}$. The flap splits into anterior and posterior halves or the entire muscle utilized in the reconstruction.

The use of temporalis flap for reconstruction of ablative defects within the maxillofacial region in 1898, with a report by Glovine on its use of it for the obliteration of dead space following orbital exenteration and primary maxillary reconstruction after cancer excision Gillies reported the use of the temporalis muscle in the reanimation of the paralyzed face.

In 2002, Abubakar and colleagues did a study in Virginia, USA on the Temporalis muscle flap in reconstruction of intraoral defects ${ }^{4}$. The criteria used to evaluate the results of this technique included flap necrosis, facial nerve deficit, limitation of mouth opening and cosmetic deformity from scarring of incision or loss of muscle volume in temporal fossa.
In 2002, Menderes A in Turkey did a study on the use of Temporalis muscle flap for the reconstruction of the orbital exenteration defects and found that this flap enables placement of highly vascularized tissue that provides the reconstructive goals of primary healing obliterating dead space with separation of the orbit from nasal cavity or sinuses $^{5}$.

In 2003, Balaji SM did study in India on Temporalis anchorage in crainiomandibular ankylosis and found that Temporalis muscle flap is an ideal interpositional material due to its close proximity to the site and good vascular supply ${ }^{6}$.

In 2003, Tender GC did a study in Louisiana, USA on vascularized Temporalis muscle flap for treatment of ottorrhea and found that none of the patients experienced recurrence of ottorrhea or meningitis. There was no complication related to the intracranial Temporalis muscle flap.

In 2004, Wong TY don study in Taiwan on Temporalis muscle flap for intraoral reconstruction and found that Temporalis muscle flap is simple and safe to apply, it can extend further in the posterior oral cavity and has fewer complications.

In 2005, Estelle's Ferriol JE et al did a study in Spain on Temporalis muscle flap and found that the Temporalis myofascial flap is an excellent choice for oral \& maxilla- 
facial region defects reconstruction and no total necrosis of even a single case out of 22 was noted. ${ }^{7}$

In 2005, Smith JE et al did a study in the Dallas USA on the utility of Temporalis muscle flap for oropharyngeal base of tongue and nasopharyngeal reconstruction.

Methods to facilitate monitoring of the flap vary greatly in complexity and invasiveness. Assessment of flap begins with a clinical evaluation of temperature, color, capillary refill and bleeding with puncture. The flap and surrounding tissue assessed to identify a possible problem extrinsic to the flap, such as hematoma, a seroma, kinking or compression from positioning.

Bleeding from stab wounds can be a useful in identifying a problem and determining whether it is predominantly arterial or venous. More formal tests to assess blood flow and viability include chemical tests such as the use of vital dyes, nuclear medicine studies and use of specific sensors (e.g., oxygen pressure probes, laser Doppler, temperature probes). Fluorescence can be useful when injected intravenously at a dose of $1.5 \mathrm{mg} / \mathrm{kg}$ : an ultraviolet light used to check for fluorescence at approximately 20 minutes. Fluorescence has the disadvantages of requiring at least 20 minutes performing, being able to assess only the exposed portion of the flap, being able to repeat only every 8 hours, being associated occasionally with nausea and tending to underestimate flap perfusion. Instruments are available to quantify the amount of fluorescein in the tissue, allowing administration of smaller doses and more frequently assessment of flap viability.

Basic Principles of Flaps

- $\quad$ Plan \& Design.

- Cannot violate its blood supply.

- Should generously fit the wound.

- Ratio length: width (avg) 2:1.

- Avoid areas of tension.

- Transfer.

- Avoid kinking, compression, tension, or severe angulations.

- Always favor gravity and venous drainage.

- Positioning.

- Use always two layers of sutures.

- Support.

\section{Material and Methods}

The Descriptive study carried out at department of Oral and Maxillofacial Surgery King Edward Medical University / Mayo Hospital Lahore. This study completed in six months from 1st June 2005 to 30th Nov 2005. A total sample of 30 patients was included in this study. Patients were included in this study, who had large defect after oncologic resection, posttraumatic or congenitally. Patient who had undergone radiation therapy, previous surgery or trauma to the temporal region were not included in this study. Medically compromised patients, who could not tolerate general anesthesia, excluded from the study. The temporalis muscle ex- posed by using a coronal incision with a preauricular extension. Dissection carried out at a subgaleal level to expose the muscle. Then, it mobilized subperiosteally from its deep origin in the temporal fossa. Anteriorly, the muscle was elevated from the lateral aspect of the orbit and inferiorly down to the temporal crest. Particular care was taken to preserve the blood vessels entering from its inferior aspect. The zygomatic arch exposed to rotate the muscle into the oral cavity. The muscle pulled through the tunnel and sutured intraorally. A drain placed at the subgaleal plane, the coronal flap repositioned, and the incision closed in layers.

Type of defect could be traumatic, congenital or developed after oncologic resection. Vitality of flap assessed by color of flap (normal, pale or bluish). Suture dehiscence, marginal necrosis, presence of infection or pus checked. Another parameter was, to check mouth opening, that could be affected or not. Finally, degree of satisfaction in mastication, speech and flap esthetics documented. The collected data analyzed by SPSS statistical package version 11.0.

\section{Results}

There were total numbers of 30 patients among those 22 was males and eight were females. At the time of presentation, their mean age was 33.7 years (males 34 years and female 32 years).

Among 30 patients, 18 patients had oncologic resection, nine had trauma and three presented with congenital cleft palate. Among 18 patients who had previous oncologic resection included 11 with squamous cell carcinoma of oral cavity, 5 with adenoid cystic carcinoma of palate and 2 with adenocarcinoma of palate. Nine patients who were posttraumatic included four patients with history of firearm injury, 3 patients had oroantral fistula because of trauma and two patients had road traffic accident.

Among the patients with congenital cleft palate, one had prognathic mandible with classIII occlusion. She had also supernumerary teeth in both arches.

Vitality of flap checked by color of flap. Color of flap remained normal in 24 patients, but it became bluish in four patients and pale in two patients. In one patient, color become normal from bluish but the rest of three patients had persistent bluish color. Margins of flap remained intact in 27 patients while in three patients, they lost their contact with recipient tissue. Twenty-seven patients had no dehiscence of suture, while three patients showed its presence. Flap also got into marginal necrosis and tissue loss in two patients. In the rest of 28 patients, there was no marginal necrosis and tissue loss. Three flaps developed infection and there was discharge of pus. Rest of 27 flaps had not developed any sign of inflammation or infection. Temporalis muscle is one of the muscles of mastication, so effects on mouth opening were also determined. In 25 patients, mouth opening remained normal; five patients had developed limited mouth opening initially, which became normal after undertaking exercises. Among 30 patients, 15 were fully satisfied with mastication after the use of temporalis muscle flap. Ten 
patients were moderately satisfied but five patients were unsatisfied with mastication after reconstruction.

Among the 30 patients, 18 patients had improvement in their speech and they were fully satisfied for their speech. Eight patients were moderately satisfied but four patients were unsatisfied for their speech. Twenty patients were fully satisfied with esthetics of flap, five patients were moderately satisfied but five patients were unsatisfied with flap esthetics.

\section{Discussion}

The same surgeons did all the flap surgeries to reduce bias. In oncological resection cases, tumors such as squamous cell carcinoma of cheek, adenoid cystic carcinoma of palate and adenocarcinoma of palate were included.

We have determined the vitality of flap by observing color of flap. A flap that had an intact blood supply with a good perfusion showed normal color, while a pale looking or cyanosed flap warranted a poor blood perfusion. Blood supply compromised in case of edema. This may initially lead into pale color and then gradually bluish color, which shows a receding blood supply. Among 30 patients, 24 patients had normal color, which shows that they had normal blood supply. However, in four patients, flap color was bluish. Conservative measures like release of pressure dressing and maintenance of negative suction drainage to prevent hematoma formation improved the condition of these flaps. Within the first 24 hours, one patient's flap was improved. However, three patients showed persistent bluish color of flaps. Two others patients showed pale colored flaps. The success rate of the flap was $90 \%$ in this study. Ten percent flaps culminated in necrosis, giving a $10 \%$ failure rate. Abubakar in 2002 conducted study on similar lines ${ }^{4}$. His sample consisted of eight patients. He reported $100 \%$ success rate. The $10 \%$ failure rate in our study was mainly because these $10 \%(\mathrm{n}=3)$ patients were diabetic and were of old age.

An increase susceptibility to infection due to compromised blood supply and due to a lowered status of immunity contributed to failure of three flaps.

Temporalis muscle used in post-oncological resection cases, in a study done by Hanasono in $2001^{8}$. No failure of flap found in a sample of nine patients. However, in our study the reason for flap failure was uncontrolled diabetes mellitus.

The presence of marginal necrosis used as a subjective corroborative measure of flap success. Marginal necrosis ensues with dwindling blood supply at the distal portions of the flap. Only two patients (6.66\%) out of a total 30 showed signs of edge necrosis with simultaneous suture dehiscence and variable tissue loss.

Temporalis muscle showed effects on mouth opening of patients of maxillofacial defects in this study. Twenty-eight patients had no effect on mouth opening after its use but only two patients developed limited mouth opening. Due to defect close to the palatoglossal arch area, limitation of mouth opening noted as possible complication. Only two patients (6.66\%) experienced a variable degree of limitation in mouth opening.

Fifty percent patients were fully satisfied with their mastication. 33.33\% of patients described their rehabilitation of mastication as moderately satisfactory while $16.67 \%$ expressed dissatisfaction with rehabilitation of masticatory functions. In that $16.67 \%$, patient's common denominator had maxillectomy and hence this attributed to dissatisfaction with the masticatory function.

The rehabilitation of speech used as a subjective measure in restoration of function and an important contributor towards the overall rehabilitation of the patients. Sixty percent patients were fully satisfied, while $26.66 \%$ were moderately satisfied. $13.34 \%$ patients showed displeasure with their speech restoration and categorized as dissatisfaction. The reason for this was not discernable.

To know about esthetics of donor site after use of these flap $66.66 \%$ patients were fully satisfied while $16.66 \%$ were moderately satisfied and same number of patients was unsatisfied. However, this study shows less satisfaction as compared to international studies.

Abubakar AO noted less complication rates than this study, the reason might be a reduced number of patients, as in his study, eight patients were included while in our study 30 patients were included.

Temporalis muscle flap is a useful, reliable option for reconstruction of moderate to large size defects. The muscle can provide abundant tissue with minimal to no functional morbidity and it is only possible if we adhere to basic principles of reconstruction.

\section{Conclusions}

Temporalis muscle flap is an axial pattern flap, which is an excellent choice for oral and maxillofacial reconstruction, especially defects of palate and cheek. Donor site esthetics was accepted by patients and offer great flexibility and shows good results in ablative surgery. It is advocated that temporalis muscle flap be considered as a primarily means of reconstruction of palatal and buccal defects. Temporalis muscle flap is far less bulky, more pliable, non-hair bearing and in close proximity to the oral cavity. Use of this muscle flap does not impair mandibular function. The depression is minimal. Hair covers most of this donor area.

\section{References}

1. Mani V, Panda AK. Versatility of temporalis myofascial flap in maxillofacial reconstruction--analysis of 30 cases. Int J Oral Maxillofac Surg 2003; 32: 368-72.

2. Birt BD, Antonyshyn O, Gruss JS. The temporalis muscle flap for head and neck reconstruction. J Otolaryngol. 1987; 16: 179-84.

3. Booth PW. Reconstructive surgery- major flaps. In: Langdon JD, Patel MF eds. Operative Maxillofacial Surgery. Cambridge: Chapman \& Hall, 1998: 83-91. 
4. Abubaker $\mathrm{O}$ and Abouzgia MB. The temporalis muscle flap in reconstruction of intraoral defects: An appraisal of the technique. Oral Surg, Oral Med, Oral Pathol, Oral Radiol and Endodon 2002; 9.

5. Menderes A, Yilmaz M, Vayvada H. Reverse temporalis muscle flap for the reconstruction of orbital exenteration defects. Ann Plast Surg 2002; 48: 521-6.

6. Balagi SM. Modified temporalis anchorage in craniomandibular reankylosis. Int J Oral Maxillofac Surg
2003; 32: 480-5.

7. Estelles Ferriol JE, Carrasco Llatas M, Ferrer Ramirez MJ, et al. Temporalis myofascial flap: technique description and results in our patients. Acta Otorrinolaringol Esp 2005; 56: 257-60.

8. Hanasono MM, Utley DS, Goode RL. The temporalis muscle flap for reconstruction after head and neck oncologic surgery. Laryngoscope 2001; 111: 1719-25. 